02

\title{
Спектры поглощения пробного сигнала и резонансной флуоресценции для излучателей при их взаимодействии с локальным окружением в прозрачных средах
}

\author{
(C) Е.А. Смирнова ${ }^{1,2,3}$, Н.А. Лозинг $\Gamma^{1,2,3}$, М.Г. Гладуш ${ }^{1,3}$, А.В. Наумов ${ }^{1,3,4}$ \\ ${ }^{1}$ Институт спектроскопии РАН, \\ 108840 Москва, Россия \\ ${ }^{2}$ Национальный исследовательский университет „Высшая школа экономики“, \\ 101100 Москва, Россия \\ ${ }^{3}$ Московский педагогический государственный университет, \\ 119435 Москва, Россия \\ ${ }^{4}$ Троицкое обособленное подразделение ФИАН, \\ 142190 Москва, Россия \\ e-mail: ksmirno@yandex.ru, Lozing@phystech.edu, mglad@isan.troitsk.ru
}

Поступила в редакцию 09.07.2021 г.

В окончательной редакции 20.09.2021 г.

Принята к публикации 25.09.2021 г.

\begin{abstract}
Представлен теоретический анализ двух типов спектральных зависимостей для нескольких конфигураций систем двухуровневых излучателей света с учетом влияния на них локальных полей и близкого окружения внутри прозрачной среды. Произведен расчет спектров поглощения слабого пробного сигнала и спектров резонансной флуоресценции при непрерывном возбуждении сильным монохроматическим полем (непрерывным лазерным излучением). Произведено сравнение чувствительности методов абсорбционной и эмиссионной оптической спектроскопии для выявления эффектов влияния среды на отдельные излучатели и их ансамбли. Спектральные зависимости были рассчитаны для модельных излучателей с учетом влияния локальных полей в прозрачной диэлектрической среде и локальных электрон-фононных взаимодействий, которые определяли реакцию излучателей на внешнее поле и эффективные механизмы релаксации. Вычислительный формализм основан на полуклассическом подходе, релаксационные процессы, связанные с фононным вкладом, вводились с учетом результатов более ранних исследований.
\end{abstract}

Ключевые слова: спектр поглощения, резонансная флуоресценция, метод пробного поля, квантовая точка, электрон-фононное взаимодействие, локальное поле.

DOI: $10.21883 / \mathrm{OS} .2022 .01 .51903 .36-21$

\section{1. Введение}

В современных исследованиях и приложениях свет может служить источником информации об испускающем его объекте и, наоборот, быть инструментом целевого или селективного воздействия на объект. В обоих случаях необходимо учитывать светозависимые процессы, развивающиеся на макро-, микро- и наномасштабах. Поглощение света отдельными частицами и их ансамблями с последующим испусканием вторичного излучения (фотолюминесценции) объединяет эти вопросы в рамках одной задачи. При этом отдельный интерес представляют излучатели-примеси. Внедрение фотоактивных частиц в прозрачный материал обеспечивает стабильное пространственное распределение квантовых излучателей и, как следствие, определенный характер производимой ими фотолюминесценции. При этом известно, что характеристики вторичного излучения определяются комбинацией свойств среды-носителя и самих частиц. Некоторые типы излучателей-примесей могут демонстрировать явление резонансной флуоресценции, т. е. ситуации, когда фотолюминесценция обеспечивается всего двумя состояниями квантового излучателя. В качестве двухуровневых излучателей могут безусловно выступать атомы в поле лазерной волны, близкой по частоте к отдельному атомному переходу. Резонансная флуоресценция независимых одиночных частиц должна демонстрировать яркие отличительные свойства, среди которых, в первую очередь, эффекты антигруппировки фотонов и трехпиковый спектр испускания $[1,2]$. Именно эти признаки также многократно наблюдались для некоторых органических молекул [3], полупроводниковых квантовых точек [4,5] и центров окраски в алмазах [6]. Таким образом, резонансная флуоресценция присуща многим типам квантовых излучателей при соблюдении ряда специфических условий. При этом важно отметить, что все указанные альтернативные природным атомам квантовые частицы могут взаимодействовать со светом в режиме двухуровневого излучателя именно в условиях, когда излучатель находится внутри среды-носителя. Современные технологии позволяют зафиксировать частицы в твердотельных матрицах или на поверхностях твердых тел. Однако расположение отдельных частиц внутри среды делает их неодинаковыми по одному или 
нескольким параметрам за счет неоднородностей носителя и наличию локальных условий. Поэтому конкретное расположение излучателя определяет, какой свет он излучает. Это, с одной стороны, делает ансамбли излучателей неоднородными, а с другой - позволяет использовать такие излучатели в качестве сенсоров, детектирующих различные явления и состояния среды на разных масштабах. Последнюю задачу можно решить с помощью анализа данных, полученных методами оптической спектроскопии. При этом оптическая спектроскопия позволяет изучать свойства квантовых систем, а для некоторых структур материалов может быть единственным доступным типом измерений. В данной работе представлен расчетно-теоретический анализ спектров резонансной флуоресценции и спектров поглощения пробного поля для различных двухуровневых излучателей с разными каналами взаимодействия между собой и средой. В данном исследовании рассматриваются спектры для независимого набора одиночных двухуровневых излучателей, коллективного ансамбля взаимодействующих двухуровневых излучателей, независимого набора одиночных модельных квантовых точек с учетом электрон-фононного взаимодействия, а также ансамбля таких модельных квантовых точек.

Явление резонансной флуоресценции имеет обобщения на более сложные случаи, т.е. может быть актуальным для излучателей с системой многих уровней и наличием более одного резонансного перехода [79]. Вместе с этим спектр резонансной флуоресценции всегда специфичен, что используется для определения наличия самого явления. В пределе „сильного поля“", т.е. в условиях насыщения переходов или близко к нему, наблюдатель будет фиксировать наличие дополнительных максимумов относительно спектра поглощения или естественного контура испускания. Это явление также известно как динамический эффект Штарка [10] и формирование „одетых атомных состояний“ [11]. Таким образом, неупругая компонента резонансной флуоресценции двухуровневого излучателя имеет трехпиковую структуру, известную как триплет Апанасевича-Моллоу [12,13]. В триплете присутствуют центральный пик на частоте возбуждающего лазера (рэлеевской компоненты рассеяния) и два сателлита, отстроенных в области меньших и больших частот. Частотные расстояния между пиками, отношения их высот и ширин определяются эффективными значениями параметров перехода излучателя, возбуждающего света, процессами дефазировки и энергетического обмена с матрицей носителя. Специфические спектры резонансной флуоресценции впервые наблюдались в эксперименте с коллимированным пучком атомов натрия [1]. За этим наблюдением последовали многочисленные эксперименты с атомарными газами, которые полностью подтвердили более ранние теоретические описания и подтвердили, что атомы могут вести себя как „идеальные“ двухуровневые излучатели, включая проявление эффекта антигруппировки фотонов [2]. Потребовалось несколько десятилетий, чтобы сделать возможным наблюдение резонансной флуоресценции с излучателями иных типов. В настоящее время резонансная флуоресценция в видимом диапазоне успешно наблюдается на одиночных молекулах [3] и полупроводниковых квантовых точках $[4,5,14]$. Органические молекулы также могут рассматриваться как двухуровневые системы при взаимодействии друг с другом. Это наблюдается в экспериментах по регистрации фотолюминесценции от пары близко расположенных молекул с дипольдипольным взаимодействием [15]. В таком случае спутанная кооперативная система будет обладать четырьмя энергетическими состояниями и сложным спектром флуоресценции [3]. При этом спектр возбуждения фотолюминесценции будет отражать факт возбуждения трех коллективных состояний и образовывать кооперативный триплет. Аналогично анализу триплета Моллоу состояние излучателей и их окружения можно восстанавливать по характеристикам трех максимумов в спектральной зависимости. При этом необходимо отметить, что триплет фотолюминесценции двухуровневой частицы и триплет возбуждения фотолюминесценции кооперативной пары двухуровневых частиц объединяет спектральная эквидистантность максимумов и наличие закономерностей, определяющих соотношения их высот и ширин.

Помимо регистрации спектров испускания и возбуждения фотолюминесценции или определения статистики фотонов от двухуровневых излучателей, также может оказаться полезным изучить эффективную двухуровневую среду под воздействием двух перестраиваемых лазеров. В этом случае один из лазеров будет обеспечивать накачку излучателей с регулируемой мощностью и задаваемой отстройкой от резонанса. Второй луч представляет сканирующий пробный сигнал от перестраиваемой лазерной системы малой мощности. Лазер накачки создает „одетую“ систему света и двухуровневой системы для определенной мощности и расстройки. Затем производится измерение поглощения или усиления пробного сигнала в зависимости от его частоты (длины волны). Данная функция обеспечит информацию о структуре одетых состояний $[11,16]$, созданных лазером накачки. На частотах, соответствующих положению сателлитов триплета Моллоу, пробный сигнал будет или максимально поглощаться, или усиливаться в зависимости от знака отстройки накачки от „атомного“ перехода. Усиление пробного поля происходит за счет энергии лазера накачки [16]. Положение полос поглощения и усиления, в свою очередь, можно регулировать, изменяя мощность накачки. Комбинации данных спектроскопических техник предлагаются в этой статье в качестве инструментов для определения двухуровневого поведения реальных излучателей и изучения влияния релаксационных процессов, кооперативных эффектов и взаимодействий с фононным резервуаром на их абсорбционные и эмиссионные свойства. Метод спектроскопии поглощения пробного поля будет реализован теоретически для вычисления спектров поглощения слабого пробного 
сигнала и спектров резонансной флуоресценции. Данный метод расчетов спектральных характеристик излучения относится к полностью полуклассическим подходам при наличии известного управляющего уравнения для матрицы плотности излучателя.

\section{2. Управляющее уравнение для излучателя и уравнения для спектральных характеристик}

Для вычисления характеристик испускаемого света необходимо определить характер работы материальной системы, являющейся источником света и поглотителем внешнего излучения. В качестве основы рассмотрим ансамбль двухуровневых излучателей, распределенный в материале, который слабо поглощает падающий внешний свет. Материал-носитель будем описывать как сплошную среду, заполняющую пространство между излучателями и характеризуемую комплексной функцией диэлектрической проницаемости. В первом приближении при построении модели предположим, что ансамбль представляет набор идентичных частиц, равномерно распределенных в объеме образца Для отдельной частицы в таком ансамбле управляющее уравнение для ее матрицы плотности $\rho$ было получено в [17-19]. Вывод данного материального уравнения не требовал применения феноменологических процедур и был произведен в рамках многочастичного квантово-кинетического формализма на основе боголюбовских цепочек редуцированных матриц плотности и корреляционных операторов. В настоящем анализе это управляющее уравнение используется для введения и анализа слагаемых, описывающих влияние окружающей среды на излучатель. Согласно [19], в общем виде в представлении взаимодействия для матрицы плотности $\rho_{I}$ можно записать

$$
\begin{aligned}
i \frac{d}{d t} \rho_{I}= & -\frac{l(\epsilon)}{\hbar}\left[\hat{\mathbf{d}}_{I}\left(\mathbf{E}+\int_{0}^{t} d t^{\prime} \sum_{a^{\prime} \neq a} \overleftrightarrow{\mathbf{G}}_{a a^{\prime}} \mathbf{p}_{a^{\prime}}\right), \rho_{I}\right] \\
& -\frac{l(\epsilon)}{\hbar}\left[\hat{\mathbf{d}}_{I}, \int_{0}^{t} d t^{\prime}\left(\overleftrightarrow{\mathbf{G}}_{a a}^{+} \hat{\mathbf{d}}_{I} \rho_{I}+\overleftrightarrow{\mathbf{G}}_{a a}^{-} \rho_{I} \hat{\mathbf{d}}_{I}\right)\right]
\end{aligned}
$$

Здесь $\quad \hat{\mathbf{d}}_{I}=\mathbf{f}(t) \hat{\sigma}^{+}+\mathbf{f}^{*}(t) \hat{\sigma}^{-}-$оператор дипольного момента перехода, в котором $\mathbf{f}(t)=\mathbf{u} \mu \exp \left(i \omega_{0} t\right)$, а $\hat{\sigma}^{+}$ и $\hat{\sigma}^{-}$- атомные повышающий и понижающий операторы. Функция $\mathbf{f}(t)$ состоит из единичного вектора в направлении диполя $\mathbf{u}$, матричного элемента для электродипольного перехода $\mu$ и временной зависимости с частотой перехода $\omega_{0}=\left(E_{2}-E_{1}\right) / \hbar$, где $E_{1}$ и $E_{2}$ - энергии основного и возбужденного состояний излучателя соответственно. Первая часть поля $\mathbf{E}=\mathbf{E}\left(\mathbf{r}_{a}, t\right)$ - это решение для плоской монохроматической волны в среде с диэлектрической проницаемостью $\epsilon$ в точке $\mathbf{r}_{a}$, соответствующей положению излучателя. Обозначение $\overleftrightarrow{\mathbf{G}_{a a^{\prime}}}=\overleftrightarrow{\mathbf{G}}\left(\mathbf{r}_{a}-\mathbf{r}_{a^{\prime}}, t-t^{\prime}\right)$ coответствует тензору Грина для поля в этой среде с верхними индексами \pm , соответствующим опережающей и запаздывающей компоненте тензора соответственно, a $\overleftrightarrow{\mathbf{G}}_{a a}^{ \pm}=\overleftrightarrow{\mathbf{G}}_{a a}^{ \pm}\left(\mathbf{r}_{a}-\mathbf{r}_{a}, t-t^{\prime}\right)=\overleftrightarrow{\mathbf{G}}^{ \pm}\left(0, t-t^{\prime}\right)$. Неоператорные величины $\mathbf{p}_{a^{\prime}}=\langle\hat{\mathbf{d}}\rangle_{a^{\prime}}$ - это индуцированные дипольные моменты остальных излучателей в ансамбле, где $\langle>$ обозначает квантово-механическое среднее. Функция $l(\epsilon)$ - это коэффициент коррекции локального поля, создаваемого матрицей-носителем, который равен $l(\epsilon)=1$ для $\epsilon=1$. Эта функция принимает одну из явных форм в зависимости от модели, выбранной для оценки локального поля, вносимого матрицейносителем [17]. Остальные обозначения являются стандартными и относятся ко времени, мнимой единице, постоянной Планка и коммутатору операторов. В данной работе уравнение (1) будет базовым для вывода всех последующих уравнений для рассматриваемых систем. Комментарии к его слагаемым будут приводиться далее по мере необходимости. В общем виде при разрешении интегрирования по времени и суммирования по частицам ансамбля в приближении вращающейся волны (1) обеспечит описание матрицы плотности излучателя с учетом локальных полей, вносимых ансамблем и сплошной средой, и эффективных скоростей радиационной релаксации. Таким образом, все известные параметры материальных уравнений, такие как сдвиги собственных частот, скорости накачки и релаксаций, будут зависеть от параметров среды-носителя. Для целей настоящей работы представляет интерес возможность таких изменений без обсуждения специфики конкретных экспериментальных ситуаций.

Метод расчета спектров в рамках полуклассического описания может быть объяснен на примере простейшего случая управляющего уравнения Линдблада или оптических уравнений Блоха. Для расчета излучения и поглощения системами с более сложным управляющим уравнением потребуются похожие действия с большим объемом вычислений. Покажем, что уравнение (1) при отсутствии среды и других излучателей приводит в точности к базовым уравнениям для двухуровневого атома с непрерывной накачкой. Для случая единственного излучателя в ансамбле, расположенного в точке $\mathbf{r}_{a}$, слагаемое с суммой в первом коммутаторе исчезает. Применив марковское приближение для матрицы плотности в подынтегральном выражении во втором коммутаторе, можно легко выполнить интегрирование по времени. В приближении вращающейся волны это слагаемое дает радиационную релаксацию и радиационный сдвиг частоты перехода. Два вклада в уравнение определены соответственно мнимой и действительной частями выражения. Поскольку обычно предполагается, что частотный сдвиг мал, то достаточно оставить только мнимую часть. Затем необходимо перейти к картине Шредингера для матрицы плотности $\rho$ с помощью оператора энергии свободного излучателя $\hat{H}_{0}=\hbar \omega_{0} \sigma_{z}$, 
определяющегося оператором инверсии населенностей $\sigma_{z}=1 / 2\left[\sigma^{+}, \sigma^{-}\right]$. Оператор дипольного перехода теперь будет иметь вид $\hat{\mathbf{d}}=\mathbf{u} \mu\left(\sigma^{+}+\sigma^{-}\right)$. Кроме того, как уже было отмечено, имеет смысл временно упростить задачу, рассмотрев среду с $\epsilon=1$. Здесь и в следующих моделях поле падающего излучения на частоте $\omega$ всегда будет описываться как $\mathbf{E}_{d}=\mathbf{e} E_{d}(t)=\mathbf{e} \mathscr{E} \operatorname{Re}\{\exp (i \omega t)\}$ с амплитудой $\mathscr{E}$ и единичным вектором поляризации е. После выполнения этих шагов мы придем к известному уравнению:

$$
\begin{aligned}
i \frac{d}{d t} \rho= & \frac{1}{2} \omega_{0}\left[\sigma^{+} \sigma^{-}-\sigma^{-} \sigma^{+}, \rho\right]-\frac{1}{\hbar} \mathbf{u} \cdot \mathbf{e} \mu E(t)\left[\sigma^{+}+\sigma^{-}, \rho\right] \\
& +\frac{i}{\hbar^{2}} \mu^{2}\left(\mathbf{u} \cdot \operatorname{Im} \overleftrightarrow{\mathbf{G}}_{a a}^{\omega_{0}} \cdot \mathbf{u}\right)\left(\left[\sigma^{-}, \rho \sigma^{+}\right]-\left[\sigma^{+}, \sigma^{-} \rho\right]\right)
\end{aligned}
$$

где $\overleftrightarrow{\mathbf{G}} \omega_{a a}^{\omega_{0}}=\overleftrightarrow{\mathbf{G}}\left(\mathbf{r}_{a}-\mathbf{r}_{a}, \omega_{0}\right)=\overleftrightarrow{\mathbf{G}}\left(0, \omega_{0}\right)$. Уравнение можно переписать в более компактной и узнаваемой форме, сделав несколько преобразований и введя традиционные обозначения. Первое слагаемое в правой части определено оператором энергии свободного атома $\hat{H}_{0}$. Во втором слагаемом для индуцированных диполей получается $(\mathbf{u} \cdot \mathbf{e})=1$ и можно записать оператор взаимодействия $\hat{V}=-\hbar \Omega\left(e^{i \omega t}+e^{-i \omega t}\right)\left(\sigma^{+}+\sigma^{-}\right)$, где $\Omega=\mu \mathscr{E} / 2 \hbar-$ частота Раби. Последнее слагаемое - это радиационное затухание со скоростью $\gamma=2 \mu^{2} / \hbar \mathbf{u} \cdot \operatorname{Im} \overleftrightarrow{\mathbf{G}}\left(0, \omega_{0}\right) \cdot \mathbf{u}=$ $=4 \mu^{2} \omega_{0}^{3} / 3 \hbar c^{3}$, где $c-$ скорость света в вакууме. Операторная часть после раскрытия коммутаторов образует в точности хорошо известный оператор Линдблада $\hat{\mathscr{L}}_{\hat{O}}(\rho)=2 \hat{O} \rho \hat{O}^{\dagger}-\hat{O}^{\dagger} \hat{O} \rho-\rho \hat{O}^{\dagger} \hat{O}$, где в данном случае произвольный оператор $\hat{O}$ заменяется на оператор $\sigma^{-}$.

Уравнения (1) и (2) описывают только естественный радиационный распад, так как (1) было получено для неподвижных частиц. Однако любому примесному излучателю свойствен процесс дефазировки. Чтобы учесть это обстоятельство, (2) необходимо дополнить известным оператором $\hat{D}(\rho)=2 \sigma_{z} \rho \sigma_{z}-\sigma_{z} \sigma_{z} \rho-\rho \sigma_{z} \sigma_{z}$, где $\gamma_{\perp}-$ скорость поперечной релаксации. Определяя скорость процесса $\gamma_{\perp}$, можно формально учесть влияние всех процессов дефазировки, которые происходят в ансамблях и средах-носителях. Если собрать все релаксационные процессы в одном операторе

$$
\hat{R}(\rho)=\frac{\gamma}{2} \hat{\mathscr{L}}_{\sigma^{-}}(\rho)+\gamma_{\perp} \hat{D}(\rho)
$$

то управляющее уравнение преобразуется к хорошо известному в литературе виду

$$
i \frac{d}{d t} \rho=\frac{1}{\hbar}\left[\hat{H}_{0}+\hat{V}, \rho\right]+i \hat{R}(\rho) .
$$

Такая запись уравнения будет удобной для описания метода спектроскопии пробного поля. Формально будет необходимо рассмотреть случай, когда внешнее поле в уравнениях (1),(2) содержит как сильную, так и слабую монохроматическую составляющие. Слабую компоненту удобно представить как $\mathbf{E}_{p}=\mathbf{e}_{p} E_{p}(t)=\mathbf{e}_{p} \mathscr{E}_{p} \operatorname{Im}\left\{\exp \left(i \omega_{p} t\right)\right\}$, для которой $\mathscr{E}_{p} \ll \mathscr{E}$. Тогда в конечном уравнении (3) должен возникнуть дополнительный коммутатор с оператором $\hat{V}_{p}=i \hbar \lambda_{p}\left(e^{i \omega_{p} t}-e^{-i \omega_{p} t}\right)\left(\sigma^{+}+\sigma^{-}\right)$. Матрицу плотности, определяющуюся новым уравнением с двумя полями, обозначим как $\varrho$. Далее будем полагать, что частота $\omega_{p}$ настроена около частоты перехода $\omega_{0}$. Коэффициент связи $\lambda_{p}=\left(\mu \mathscr{E}_{p}\right) / 2 \hbar$ определяется амплитудой слабой компоненты поля $\mathscr{E}_{p}$ и всегда очень мал по сравнению с частотой Раби, определяемой сильным полем. Поскольку этот подход представляет собой метод расчета спектра, а не проблему взаимодействия с немонохроматическим полем, предполагается, что вопросы фазы и поляризации остались за кадром. В этой конфигурации мы можем найти решение для матрицы плотности $\varrho$ в виде $\varrho=\rho+p$, где $p-$ небольшая поправка к матрице, которая подчиняется уравнению (3) для случая единственного сильного поля. Тогда уравнение для $\rho+p$ принимает вид

$$
\begin{aligned}
i \frac{d}{d t}(\rho+p)= & \frac{1}{\hbar}\left[\hat{H}_{0}+\hat{V}, \rho+p\right] \\
& +\frac{1}{\hbar}\left[\hat{V}_{p}, \rho+p\right]+i \hat{R}(\rho)+i \hat{R}(p) .
\end{aligned}
$$

Вычитая уравнение (3) из уравнения (4) и пренебрегая слагаемым второго порядка малости, т. е. $\left[\hat{V}_{p}, p\right]$, получаем уравнение для поправочной матрицы $p$ :

$$
i \frac{d}{d t} p=\frac{1}{\hbar}\left[\hat{H}_{0}+\hat{V}, p\right]+i \hat{R}(p)+\frac{1}{\hbar}\left[\hat{V}_{p}, \rho\right] .
$$

Система зацепляющихся уравнений (3) и (5) будет основой дальнейшего анализа. Прежде чем получить из них систему кинетических уравнений для компонент матриц $\rho_{k l}(t)(k, l=1,2)$ и $p_{m n}(t)(m, n=1,2)$, их необходимо привести к уравнениям с параметрами, не зависящими от времени. Это можно сделать с помощью следующих замен $\rho_{21}=r_{21} e^{-i \omega t}, \rho_{12}=r_{12} e^{i \omega t}$, которые приводят (3) к системе, вращающейся с частотой лазера, и $p_{21}=\phi_{21} e^{-i \omega_{p} t}, p_{11}=\phi_{11} e^{i v t}, p_{22}=\phi_{22} e^{i v t}$, $p_{12}=\phi_{12} e^{i\left(2 \omega-\omega_{p}\right) t}$, где $v=\omega-\omega_{p}$. После внесения этих замен можно пренебречь быстро осциллирующими членами, что приведет к окончательной системе уравнений:

$$
i \frac{d}{d t} r=\frac{1}{\hbar}\left[\hat{H}_{0}^{\prime}+\hat{V}^{\prime}, r\right]+i \hat{R}(r)
$$

$$
i \frac{d}{d t} \phi=v \phi+\frac{1}{\hbar}\left[\hat{H}_{0}^{\prime}+\hat{V}^{\prime}, \phi\right]+i \hat{R}(\phi)-i \lambda_{p}\left[\sigma^{+}, r\right],
$$

где $r$ и $\phi-$ новые матрицы, а $\hat{H}_{0}^{\prime}=\Delta \sigma_{z} \quad$ и $\hat{V}^{\prime}=-\left(\beta \sigma^{-}+\beta^{*} \sigma^{+}\right)-$составляющие гамильтониана, записанного в соответствии с приближением вращающейся волны. Часть, соответствующая свободной частице, теперь пропорциональна $\Delta=\omega_{0}-\omega$, которая описывает расстройку между частотой перехода и сильным полем. Что касается оператора взаимодействия, он 
теперь содержит множитель, который в рассматриваемом простом случае равен $\beta=\beta^{*}=\Omega$ и примет более сложный вид при других обстоятельствах.

Важно отметить, что обе матрицы $\varrho$ и $\rho$ сохраняют свои фундаментальные свойства, т.е. $\operatorname{tr}(\varrho)=\operatorname{tr}(\rho)=1$. Следовательно, поправочная матрица должна удовлетворять условию $\operatorname{tr}(p)=0$. Как легко заметить, то же самое относится к матрицам $r$ и $\phi$. Средние от операторов вычисляются согласно $\langle\hat{O}\rangle=\operatorname{tr}(\hat{O} \rho+\hat{O} p)=\langle\hat{O}\rangle_{\text {strong }}+\langle\hat{O}\rangle_{\text {weak }} . \quad$ Таким образом, вклад зондирующего сигнала в общий отклик частиц на действие внешнего поля необходимо оценивать через составляющую наведенной поляризации $\mathbf{P}_{p}=N\langle\hat{\mathbf{d}}\rangle_{\text {weak }}=\mathbf{e}_{p} N 2 \mu \operatorname{Re}\left(p_{21}\right)$.

Из уравнений Максвелла следует, что объемная удельная мощность поля, которая создает электрическую поляризацию среды, равна $\langle\mathbf{E} \cdot \partial \mathbf{P} / \partial t\rangle_{t}$, где \langle\rangle$_{t}$ обозначают усреднение по времени. Так как нам необходимо найти стационарное решение, то нужно положить $d r / d t=d \phi / d t=0$, при этом долю пробного сигнала можно рассчитать как

$$
W_{\text {weak }}=\left\langle\mathbf{E}_{p} \cdot \frac{\partial \mathbf{P}_{p}}{\partial t}\right\rangle_{t}=-N \mu \mathscr{E}_{p} \omega_{p} \operatorname{Re}\left(\phi_{21}\right) .
$$

C другой стороны, эта величина может быть также определена как изменение интенсивности сигнала на единицу длины вдоль оси распространения, т. е. можно записать

$$
N \mu \mathscr{E}_{p} \omega_{p} \operatorname{Re}\left(\phi_{21}\right)=\frac{d I_{p}}{d z}=-\alpha I_{p}
$$

где $I_{p}$ есть интенсивность пробного поля, которую можно выразить следующим образом $I_{p}=\left(c \mathscr{E}_{p}^{2}\right) / 8 \pi$. Окончательно коэффициент поглощения пробного поля на определенной частоте равен

$$
\alpha=-\frac{4 \pi}{\hbar} N \mu^{2} \frac{\omega_{p}}{c} \operatorname{Re}\left(\frac{\phi_{21}}{\lambda_{p}}\right) .
$$

Далее для нахождения $\phi_{21}$ необходимо решить уравнения (6) и (7) для стационарного случая. Для этого может быть удобным переход к новым вещественным функциям из комбинаций компонент матрицы $r$. Для этого перепишем $r$ в базисе вектора Блоха $r=1 / 2(\mathbf{I}+\mathbf{B} \cdot \sigma)$, где $\mathbf{I}-$ единичная матрица, $\mathbf{B}^{T}=\left\{r_{21}+r_{12}, r_{11}-r_{22}, i\left(r_{12}-r_{21}\right)\right\}=\{u, w, v\}$ есть вектор Блоха и $\sigma^{T}=\left\{\sigma_{1}, \sigma_{2}, \sigma_{3}\right\}-$ вектор, составленный из матриц Паули. Уравнения, которые следуют из уравнения (6), приводят к известным стационарным решениям. Для простоты мы ограничиваем задачу случаем, когда в отсутствие возмущений система переходит в свое основное состояние, в то время как истинных ограничений на введение равновесных тепловых населенностей нет. Таким образом, положим $\mathbf{B}_{e q}^{T}=\left\{0, w_{e q}, 0\right\}$, где $w_{e q}=1$.

Решения для компонент $\phi(v)$ в стационарном случае удобно записать в виде вектора $\varphi^{T}(v)=\left\{\phi_{21}, \phi_{11}, \phi_{12}\right\}$.
При этом также стоит помнить, что верно $\phi_{22}=-\phi_{11}$ в силу свойства, описанного выше. Таким образом, решение $\phi$ находится из уравнения:

$$
\mathbf{M}(v) \boldsymbol{\varphi}-\mathbf{s}=0
$$

где

$$
\mathbf{M}(v)=\left[\begin{array}{ccc}
v+\Delta-i \gamma_{2} & -2 \beta^{*} & 0 \\
-\beta & v-i \gamma & \beta^{*} \\
0 & 2 \beta & v-\Delta-i \gamma_{2}
\end{array}\right]
$$

и вектор $\mathbf{s}$ определяется неоднородностью $i \lambda_{p}\left[\sigma^{+}, r\right]$. Скорость затухания $\gamma_{2}$ может описывать как дефазировку $\gamma_{2}=\gamma_{\perp}$, так и $\gamma_{2}=\gamma / 2$ в случае чистого радиационного затухания. Вектор s через компоненты вектора Блоха записывается следующим образом:

$$
\mathbf{s}=\frac{i}{2}\left[\begin{array}{c}
2 w \\
u+i v \\
0
\end{array}\right]
$$

Теперь коэффициент поглощения пробного сигнала (10) находится из

$$
\alpha(v)=-\frac{4 \pi}{\hbar} N \mu^{2} \frac{\omega_{p}}{c} \operatorname{Re}\left(\mathbf{M}^{-1}(v) \mathbf{s}\right)_{1} .
$$

Чтобы получить спектр мощности испускания (флуоресценции), нужно преобразовать неоднородный член в уравнении (7). Таким образом, здесь при определении мощности излучения будем следовать концепции, разработанной и описанной в [16]. Действительно, работу пробного сигнала следует рассматривать применительно к переходам в управляемой квантовой системе, описываемой матрицей $r$. Если „вручную“ исключить роль основного состояния из последнего слагаемого в уравнении (7) и ввести новое решение в уравнение (8), то последнее, взятое с противоположным знаком, должно описывать удельную мощность, передаваемую полю от излучателей. Поскольку передаваемая мощность содержит как упругую составляющую, т. е. чистое релеевское рассеяние внешнего поля, так и неупругую составляющую, нам будет интересно заранее исключить упругую часть. Эти „искусственные“ процедуры выглядят следующим образом:

$$
i \lambda_{p}\left[\sigma^{+}, r\right] \rightarrow-i \lambda_{p}\left(r \sigma^{+}-r\left\langle\sigma^{+}\right\rangle\right) .
$$

Это означает, что берется в рассмотрение только часть коммутатора в неоднородном слагаемом уравнения (7), но при этом также заменяется повышающий оператор его разностью со своим средним значением. В [20] правомерность такого вычитания была доказана строго и подробно в рамках полностью кваново-механического описания. Наконец, собрав вместе (7), (8), (10), (11) и (14), приходим к новому вектору:

$$
\mathbf{s}_{f}=-\frac{i}{2} \lambda_{p}^{2}\left(\left[\begin{array}{c}
1-w \\
u+i v \\
0
\end{array}\right]+(u+i v)\left[\begin{array}{c}
u-i v \\
1+w \\
u+i v
\end{array}\right]\right),
$$


который необходимо использовать для расчета доли вклада одного излучателя в передаваемую полю мощность:

$$
W_{f}(v)=2 \hbar \omega_{p} \operatorname{Re}\left(\mathbf{M}^{-1}(v) \mathbf{s}_{f}\right)_{1} .
$$

Уравнения (13) и (16) являются окончательными формулами для расчета функций, которые описывают характеристики поглощения и излучения в настоящем исследовании. Результаты расчетов спектральных зависимостей для одиночного излучателя без окружения представлены на рис. 1, $a$ и 2, $a$ соответственно. Все зависимости $\alpha(v)$ и $W_{f}(v)$ представлены в относительных единицах (размерных коэффициентах), при этом все параметры выражены в единицах скорости радиационного затухания $\gamma$. На рис. 1, $a$ представлены спектры поглощения для трех комбинаций отстройки сильного возбуждающего поля от частоты перехода в излучателе и величины управляющей частоты Раби. Для аналогичных условий были рассчитаны триплеты Моллоу на рис. 2, $a$. Из сравнения рисунков следует, что обе спектроскопические техники могут быть эффективными и информативными для анализа фотолюминесценции двухуровневых излучателей. Если экспериментальное наблюдение триплета Моллоу требует наличия спектральных устройств и техники высокого разрешения, то для спектроскопии пробного поля необходимо определять полосы поглощения и усиления путем перестройки частоты пробного лазера. Вклады различных дополнительных процессов, влияющих на излучатель, можно устанавливать из спектральных положений, относительных величин и ширин спектральных пиков.

\section{3. Модификации спектров двухуровневых излучателей}

Рассмотрим, как некоторые типы взаимодействий излучателя со своим локальным окружением в матрице носителя могут модифицировать контуры спектральных зависимостей. Из наиболее сильного влияния на оптические свойства частиц стоит выделить влияние локальных полей и локальных колебательных мод. При этом локальные поля обусловлены диполь-дипольным взаимодействием излучателя с другими излучателями и компонентами среды. Также сплошная среда накладывает условия на структуру поля, что приводит к проявлению эффекта Парсела [17,21]. При этом указанные эффекты могут обеспечивать обратную связь между эффективной накачкой, затуханием и состоянием излучателя.

Вернемся теперь к уравнению (1) и воспроизведем все преобразования для системы из $N$ излучателей в единице объема, расположенных на расстояниях, меньших длины волны возбуждения/испускания. В плотном однородном ансамбле, возбуждаемом лазерным светом, каждый атом фактически находится под действием эффективного (лоренцева) локального поля $\mathbf{E}_{\mathrm{L}}$, которое состоит из внешнего поля $\mathbf{E}$ и макроскопической поляризации $\mathbf{P}$.
Это поле может быть записано через элементы матрицы плотности [20-22]

$$
\mathbf{E}_{\mathrm{L}}=\mathbf{E}+\frac{4 \pi}{3} \mathbf{P}=\mathbf{E}+\frac{4 \pi}{3} N \mathbf{u} \mu\left(\rho_{12}+\rho_{21}\right),
$$

где $N$ - концентрация излучателей. С учетом эффективного значения $\mathbf{E}$ мы получаем уравнение (6), где в матрице $\mathbf{M}$ величина $\beta$ принимает эффективное значение:

$$
\beta=\Omega+\left(\varepsilon+\frac{i \gamma_{c}}{2}\right)(u-i v) .
$$

Здесь $\varepsilon=4 \pi N \mu^{2} / 3 \hbar$ есть так называемый красный сдвиг Лоренца и $\gamma_{c}=N \gamma / 2$ представляет собой скорость коллективного распада [20]. Из этого следует, что учет локального поля приводит к изменению матрицы $\mathbf{M}(v)$. Внутри нее появляются динамические слагаемые, зависящие от состояния излучателя. Иными словами, значения элементов матрицы плотности излучателя теперь определяют не только матрицы (12) и (15), но и основную матрицу (11). Из структуры последней следует, что динамические изменения $\beta$ будут приводить к сдвигам зон поглощения/усиления пробного сигнала и сдвигам сателлитов спектра резонансной флуоресценции. Однако в отличие от одиночных свободных излучателей (рис. $1, a$ и 2,a) их расстояние от центра уже не будет пропорционально частоте Раби $\beta=\Omega$. Этот эффект продемонстрирован на рис. $1, b$ и $2, b$. Видно, что в результате действия коллективных локальных полей увеличение накачки (удвоение $\Omega$ ) приводит лишь к незначительному сдвигу спектральных линий (сравните с рис. $1, a$ и $2, a)$. В то же время при одинаковой накачке увеличение красного сдвига Лоренца существенно меняет положение смещенных спектральных компонент. При этом видно, что также изменяются ширины линий и соотношения их высот. Однако можно предположить, что эффект изменения ширин будет не столь заметным по сравнению с уширениями, вносимыми дефазировкой и взаимодействием с фононными модами. Для рассматриваемых типов излучателей это является важным обстоятельством и должно оговариваться или учитываться при проведении моделирования.

Для построения спектров (13) и (16) для двухуровневых излучателей с сильной дефазировкой и учетом фононного вклада воспользуемся управляющим уравнением, полученным в работе [4]. В этой работе представлены теоретические и экспериментальные исследования резонансной флуоресценции полупроводниковых квантовых точек, которые подготавливались к работе в режиме одетых состояний двухуровневой системы. Управляющее уравнение в этом случае принимало следующий вид:

$$
\begin{aligned}
i \frac{d}{d t} \rho= & \frac{1}{\hbar}[\hat{H}+\hat{V}, \rho]+i \hat{R}(\rho)+i \gamma^{+} \hat{\mathscr{L}_{\sigma^{+}}}(\rho) \\
& +i \gamma^{-} \hat{\mathscr{L}_{\sigma^{-}}}(\rho)-i \gamma_{c d}\left(\sigma^{+} \rho \sigma^{+}+\sigma^{-} \rho \sigma^{-}\right),
\end{aligned}
$$

где $\gamma_{c d}$ есть скорость перекрестной дефазировки, которая влияет на недиагональные элементы матрицы 


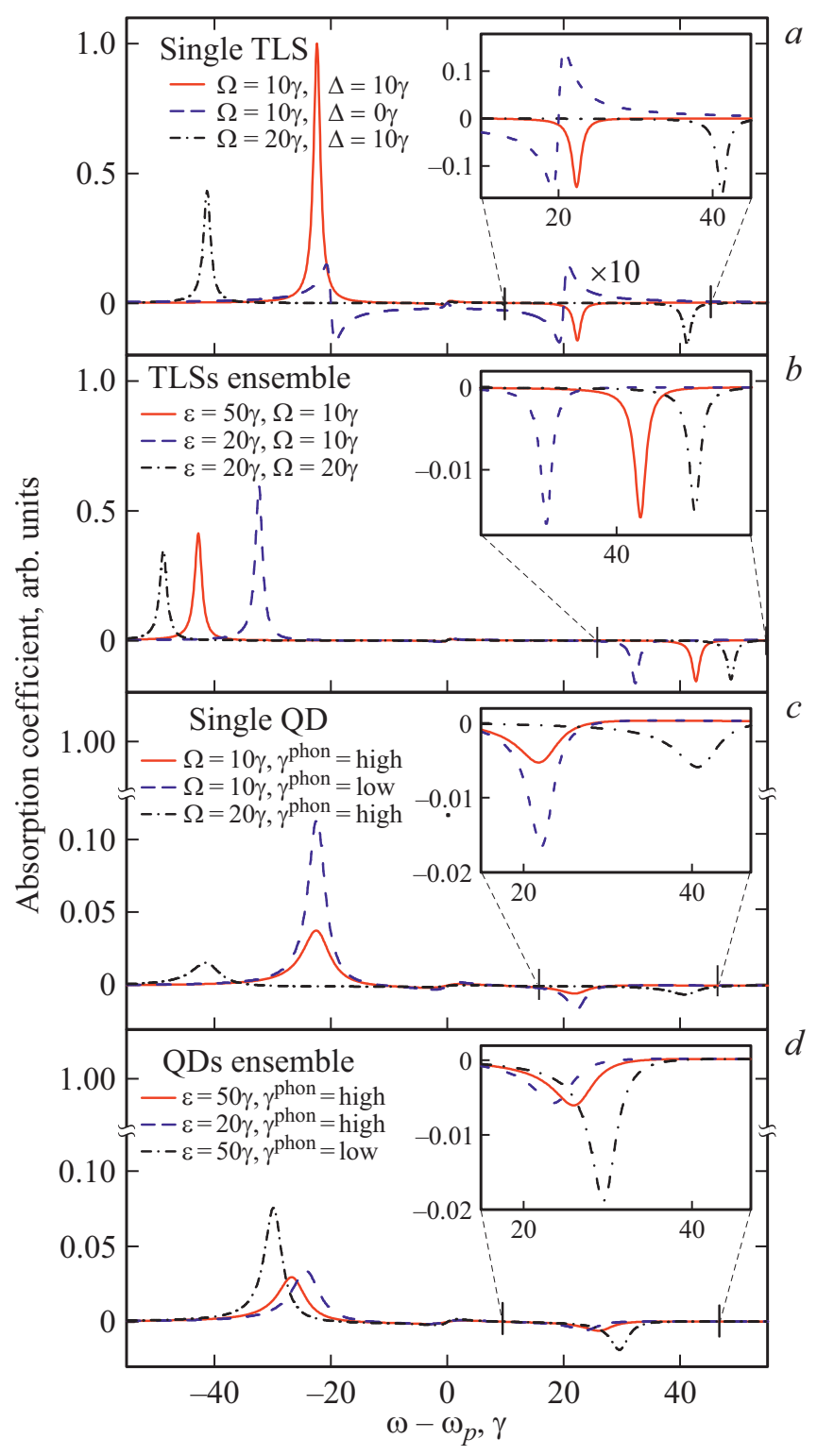

Pис. 1. Спектр поглощения слабого пробного сигнала для различных параметров: (a) одиночный двухуровневый излучатеть; $(b)$ ансамбль двухуровневых излучателей (здесь $\left.\Delta=10 \gamma, \quad \gamma_{c}=0.9 \gamma\right) ; \quad(c)$ модельная КТ (здесь $\left.\Delta=10 \gamma\right)$, где,$\gamma^{\text {phon }}=h i g h^{\text {“ }}$ обозначает набор параметров $\gamma^{+}=0.9 \gamma$, $\gamma^{-}=0.8 \gamma, \gamma^{c d}=0.6 \gamma$ и , $\gamma^{\text {phon }}=$ low“ обозначает $\gamma^{+}=0.45 \gamma$, $\gamma^{-}=0.4 \gamma, \quad \gamma^{c d}=0.3 \gamma ; \quad(d)$ ансамбль КТ (здесь $\Omega=10 \gamma$, $\left.\Delta=10 \gamma, \gamma_{c}=0.9 \gamma\right)$.

плотности, слагаемое с $\gamma^{-}$соответствует усиленному радиационному распаду, в то время как слагаемое, содержащее $\gamma^{+}$, представляет собой некогерентный процесс возбуждения. Параметры $\gamma^{ \pm}, \gamma_{c d}$ имеют медленную зависимость от расстройки с частотой лазера и могут рассматриваться как константы в пределах используемых значений частоты накачки. Таким образом, данное уравнение описывает двухуровневую систему, которая взаимодействует с источником света и фононным резервуаром, и также учитывает процессы кросс-релаксации диагональных элементов матрицы плотности. Заметим, что уравнение (18) совпадает с уравнением (3) в первых двух слагаемых. Новые слагаемые вносят изменения в матрицу М, которые представим посредством введения новой матрицы $\mathbf{M}_{p n}$, представляющей сумму матрицы, определенной в (11), и дополнительной матрицы:

$$
\begin{aligned}
& \mathbf{M}_{p n}(v)=\mathbf{M}(v)+ \\
& +\left[\begin{array}{ccc}
-i\left(\gamma^{+}+\gamma^{-}\right) & 0 & -i \gamma_{c d} \\
0 & -2 i\left(\gamma^{+}+\gamma^{-}\right) & 0 \\
-i \gamma_{c d} & 0 & -i\left(\gamma^{+}+\gamma^{-}\right)
\end{array}\right] .
\end{aligned}
$$

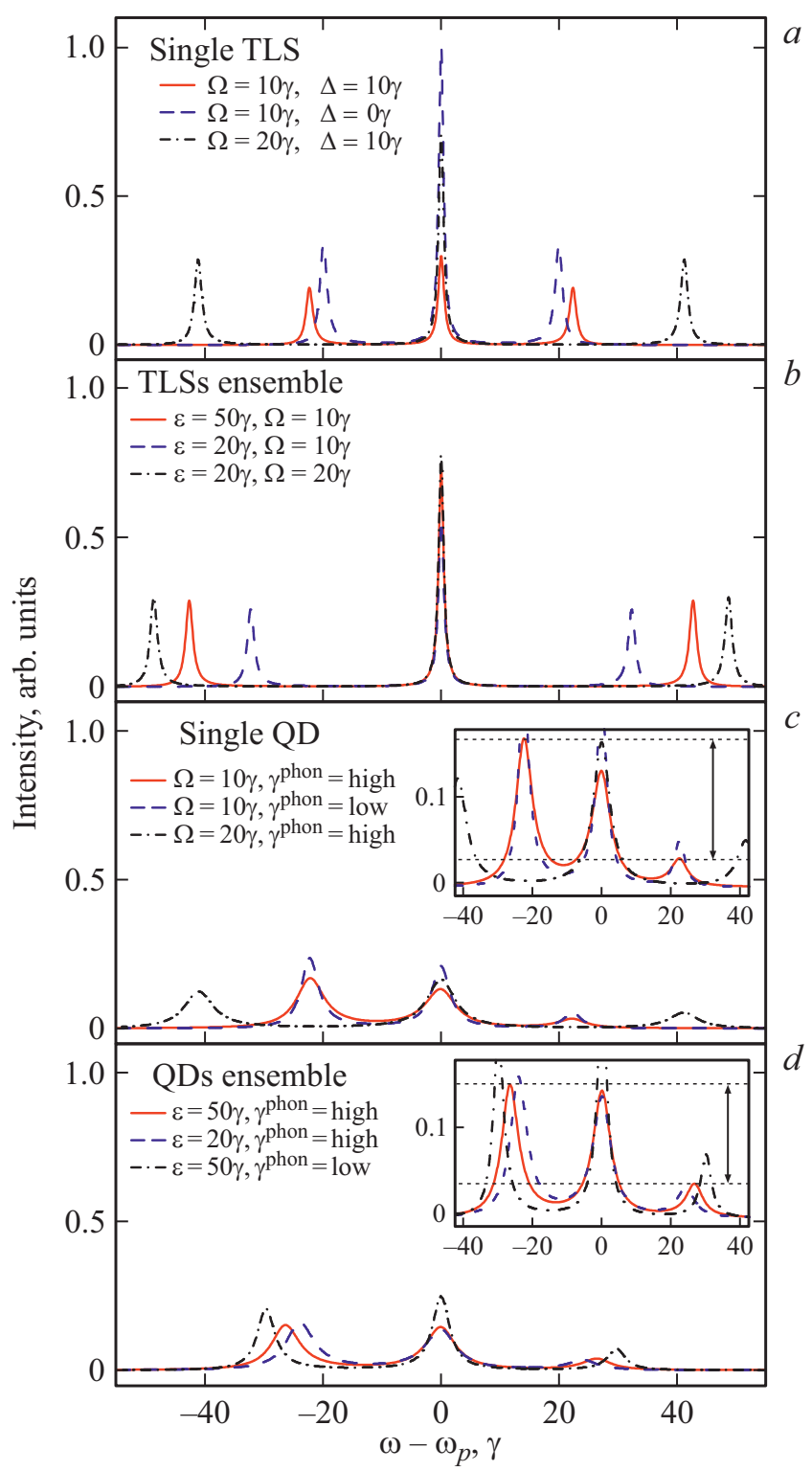

Рис. 2. Спектр флуоресценции для различных параметров: $(a)$ одиночный двухуровневый излучатель; $(b)$ ансамбль двухуровневых излучателей (здесь $\left.\Delta=10, \gamma_{c}=0.9\right),(c)$ одиночная модельная КТ (здесь $\Delta=10)$, где „, $\gamma^{\text {phon }}=h i g h^{\text {“ }}$ обозначает набор параметров $\gamma^{+}=0.9, \gamma^{-}=0.8, \gamma^{c d}=0.6$ и , $\gamma^{\text {phon }}=l o w$ “ обозначает $\gamma^{+}=0.45, \gamma^{-}=0.4, \gamma^{c d}=0.3$; $(d)$ ансамбль модельных квантовых точек (здесь $\Omega=10, \Delta=10, \gamma_{c}=0.9$ ). 
Сначала рассмотрим случай, когда в матрице $\mathbf{M}$ накачка определяется только внешним полем, т.е. $\beta=\beta^{*}=\Omega$. Далее воспользуемся матрицей $\mathbf{M}_{p n}$ и произведем расчет по формулам (13) и (16). Спектры резонансной флуоресценции становятся асимметричными относительно высот сателлитов (рис. 2,c), что в точности воспроизводит результат работы [4]. При этом в данной работе приводится расчет спектров поглощения пробного поля, из которого видно явное ослабления эффекта поглощения/усиления при значительном уширении соответствующих полос. Таким образом, спектроскопия пробного поля также обладает достаточной чувствительностью к рассматриваемым эффектам и может служить альтернативной техникой изучения процессов в материале в окрестностях излучателя. При этом остается открытым вопрос о специфичности изменения спектральных кривых при наличии нескольких сильных эффектов. С целью иллюстрации подобной ситуации рассмотрим совместное влияние ансамблевых локальных полей, влияние носителя как сплошной среды и влияние дефазировки и уширения.

Для решения последней задачи следует произвести одновременный учет коллективных эффектов и эффектов дефазировки. Это можно сделать формально, если произвести вычисления для (13) и (16), используя выражения (17) и (19). На рис. 1, $d$ и 2, $d$ приведены соответствующие результаты вычислений для выбранных параметров, позволяющих произвести сравнение с предыдущими спектральными картинами. В спектрах поглощения наблюдается заметная взаимная компенсация эффектов. Полосы поглощения и усиления смещены, но занимают положения, отличные от положений для независимых излучателей. При этом, как видно, баланс поглощения и усиления может изменяться в зависимости от соотношения параметров „конкурирующих“ процессов. Аналогичная ситуация наблюдается в спектре резонансной флуоресценции. Спектральные пики смещены относительно положений, определяемых частотой $\Omega$, но изменяется соотношение высот. При этом сохраняется асимметрия относительно как высот, так и ширин спектральных линий.

\section{4. Обсуждение и заключение}

В работе представлен теоретический анализ спектров поглощения пробного поля и спектров резонансной флуоресценции двухуровневых квантовых излучателей в различных модельных условиях, когда поглощение и испускание света зависит от параметров и состояния окружающей среды. Разработан математический аппарат вычисления спектров излучения частиц в присутствии сильного внешнего поля в полуклассическом приближении на основе известного формализма - метода пробного поля. Спектр излучения может быть восстановлен из анализа коэффициента поглощения/усиления слабого пробного сигнала с перестройкой длины волны в

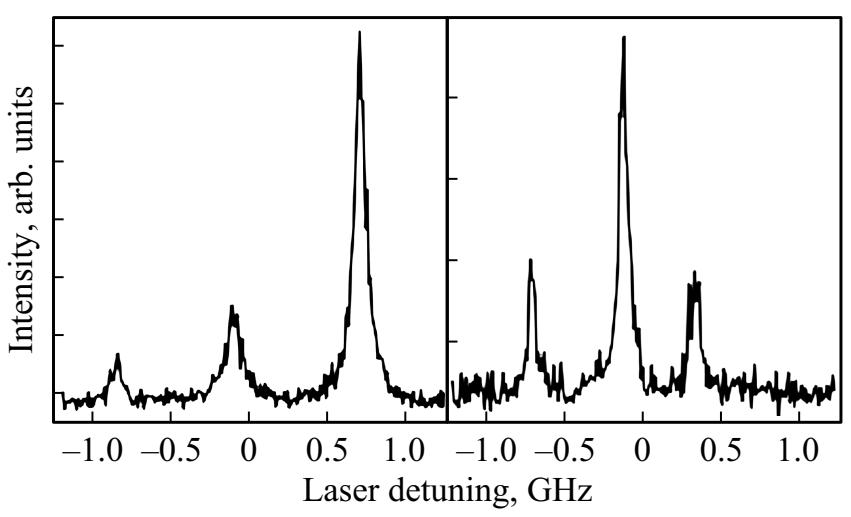

Рис. 3. Спектры возбуждения фотолюминесценции молекул терилена в тонкой пленке 1,2-орто-дихлоробензола.

окрестностях перехода излучателя, взаимодействующего с управляющим монохроматическим сигналом. Показана эквивалентность представленного метода с квантовокинетическим подходом на основе боголюбовских цепочек для матриц плотности излучателей и поля. Произведены расчеты спектров поглощения слабого зондирующего сигнала, который распространяется через среду, управляемую сильным непрерывным лазером, настроенным вблизи единственного перехода оптически активных примесных центров. Рассчитаны спектры резонансной флуоресценции для моделей, когда светоизлучающие системы испытывают влияние локальных полей и эффекты локальных электрон-фононных взаимодействий.

Из результатов проведенных расчетов следует, что рассмотренные типы спектральных зависимостей могут быть использованы для восстановления характеристик сред, содержащих зондовые частицы - излучатели. В работе анализируется явление резонансной флуоресценции двухуровневых частиц. При реализации данного типа фотолюминесценции в рассмотренных спектрах при достаточно сильной накачке присутствуют больше одной частотной полосы, характеристики которой могут содержать информацию о состоянии излучателя и его участии в процессах в среде вблизи него. Так, взаимная конфигурация параметров каждого из контуров поглощения и усиления пробного поля (частотное положение, высота, ширина) определяется параметрами накачки, наличием радиационных поправок, обусловленных средой и взаимодействием с фононными модами. Анализ зависимости спектрального контура от контролируемых параметров накачки (длина волны, мощность) и состояния среды (температура) дает возможность определять характер и типы процессов, сопутствующих фотолюминесценции. Аналогичные возможности может обеспечить анализ триплетов резонансной флуоресценции Апанасевича-Моллоу. Частотные положения, высоты и ширины пиков излучения также определяются процессами, происходящими в среде вокруг излучателя. Отдельные процессы проявляют себя однозначным образом на фоне управляемой накачки и могут быть идентифици- 
рованы. Наличие большего числа параметров (определяющих состояние единственного перехода), чем при анализе одной линии излучения, в определенных случаях может обеспечивать более точное восстановление характеристик среды. Последнее должно обеспечиваться развитием теоретических знаний об оптике примесных частиц.

В заключение необходимо отметить, что многофакторный анализ спектральных триплетов не ограничивается рассмотренными примерами и, в частности, описанными в работе возможностями использования двухуровневых излучателей. Как указывалось выше, пары двухуровневых излучателей могут быть внедрены в твердотельную среду и испытывать диполь-дипольное перепутывание. Спектры возбуждения фотолюминесценции (зависимость полной интенсивности свечения от частоты накачки) кооперативных пар также имеют форму триплета. Примеры оригинальных спектров возбуждения органических молекул представлены на рис. 3. На рисунках видно, что контуры возбуждения имеют одинаковые расстояния между пиками, но пики отличаются по высоте и ширине. Из этого следует, что сама функция контура триплета резонансной флуоресценции не является специфической для систем двухуровневых излучателей. Вместе с тем наборы характеристик спектральных максимумов в спектрах испускания и возбуждения определяются разными физическими процессами, но все подвержены влиянию локального окружения.

\section{Финансирование работы}

Работа выполнена при финансовой поддержке Российского фонда фундаментальных исследований (грант РФФИ № 19-02-00991).

\section{Конфликт интересов}

Авторы заявляют, что у них нет конфликта интересов.

\section{Список литературы}

[1] F.Y. Wu, R.E. Grove, S. Ezekeil. Phys. Rev. Lett., 35 (21), 1426 (1975). DOI: 10.1103/PhysRevLett.35.1426

[2] H.J. Kimble, M. Dagenais, L. Mandel. Phys. Rev. Lett., 39 (11), 691 (1977). DOI: 10.1103/PhysRevLett.39.691

[3] G. Wrigge, I. Gerhardt, J. Hwang, G. Zumofen, V. Sandoghdar. Nature Physics, 4 (1), 60 (2008). DOI: 10.1038/nphys 812

[4] A. Ulhaq, S. Weiler, C. Roy, S.M. Ulrich, M. Jetter, S. Hughes, P. Michler. Opt. Express, 21 (4), 4382 (2013). DOI: 10.1364/OE.21.004382

[5] Y.-J. Wei, Y. He, Y.-M. He, C.-Y. Lu, J.-W. Pan, C. Schneider, M. Kamp, S. Höfling, D.P.S. McCutcheon, A. Nazir. Phys. Rev. Lett., 113 (9), 097401 (2014). DOI: 10.1103/PhysRevLett.113.097401

[6] D. Chen, Z. Mu. Y. Zhou, J.E. Fröch, A. Rasmit, C. Diederichs, N. Zheludev, I. Aharonovich, W.-B. Gao. Phys. Rev. Lett., 123 (3), 033602 (2019).

DOI: 10.1103/PhysRevLett.123.033602
[7] R. Vlasov, A. Lemeza, M. Gladush. Laser Phys. Lett., 10 (4), 045401 (2013). DOI: 10.1088/1612-2011/10/4/045401

[8] А.А. Пантелеев, Вл.К. Рерих, А.Н. Старостин. ЖЭТФ, 117 (1), 57 (2000). [A.A. Panteleev, Vl.K. Rerikh, A.N. Starostin. JETP, 90 (1), 50 (2000). DOI: 10.1134/1.559093].

[9] А.А. Пантелеев, Вл.К. Рерих. ЖЭТФ, 119 (2), 243 (2001). [A.A. Panteleev, V1.K. Roerieh. JETP, 92 (2), 210 (2000). DOI: $10.1134 / 1.1354678]$.

[10] L. Mandel, E. Wolf. Optical Coherence and Quantum Optics, (Cambridge University Press, Cambrigde, 1995). DOI: $10.1119 / 1.18450$

[11] M.O. Scully, M.S. Zubairy. Quantum Optics, (Cambridge University Press, Cambrigde, 1997). DOI: $10.1017 / \mathrm{CBO} 9780511813993$

[12] P. Apanasevich, S.Y. Kilin. Journal of Applied Spectroscopy, 24 (4), 528 (1976). DOI: 10.1007/BF00938675

[13] B.R. Mollow. Phys. Rev., $188(5), 1969$ (1969). DOI: 10.1103/PhysRev.188.1969

[14] A. Muller, E.B. Flagg, P. Bianucci, X.Y. Wang, D.G. Deppe, W. Ma, J. Zhang, G.J. Salamo, M. Xiao, C.K. Shih. Phys. Rev. Lett., 99, 187402 (2007). DOI: $10.1103 /$ PhysRevLett.99.187402

[15] C. Hettich, C. Schmitt, J. Zitzmann, S. Kühn, I. Gerhard, V. Sandogdar. Science, 298 (5592), 385 (2002). DOI: $10.1126 /$ science. 1075606

[16] С.Г. Раутиан, Г.И. Смирнов, А.М. Шалагин. Нелинейные резонансы в спектрах атомов и молекул (Наука, Новосибирск, 1970).

[17] М.Г. Гладуш, Т.А. Аникушина, А.А. Горшелев, Т.В. Плахотник, А.В. Наумов. ЖЭТФ, $155(5), 771$ (2019). DOI: $10.1134 /$ S0044451019050018 [M.G. Gladush, T.A. Anikushina, A.A. Gorshelev, T.V. Plakhotnik, A.V. Naumov. JETP, 128 (5), 655 (2019). DOI: 10.1134/S1063776119030038].

[18] Д.В. Кузнецов, Вл.К. Рерих, М.Г. Гладуш. ТМФ, 168 (2), 261 (2011). DOI: https://doi.org/10.4213/tmf6680 [D.V. Kuznetsov, V1.K. Roerich, M.G. Gladush. Theor. Math. Phys., 168 (2), 1078 (2011). DOI: $10.1007 / \mathrm{s} 11232-011-0089-8]$.

[19] N.A. Lozing, M.G. Gladush, I.Y. Eremchev, E.A. Ekimov, A.V. Naumov. Phys. Rev. B, 102 (6), 060301 (2020). DOI: 10.1103/PhysRevB.102.060301

[20] M.G. Gladush, D.V. Kuznetsov, Vl.K. Roerich. The European Physical Journal D, 64 (2), 511 (2011). DOI: $10.1140 /$ epjd/e2011-20194-0

[21] A.V. Naumov, A.A. Gorshelev, M.G. Gladush, T.A. Anikushina, A.B. Golovanova, J. Kohler, L. Kador. Nanoletters, 18 (10), 6129 (2018). DOI: 10.1021/acs.nanolett.8b01753

[22] M. Born, E. Wolf, Principles of Optics, 7th ed. (Cambridge University Press, Cambrigde, 2013). DOI: https://doi.org/10.1017/CBO9781139644181 